\title{
FINITE LOOPS WITH NILPOTENT INNER MAPPING GROUPS ARE CENTRALLY NILPOTENT
}

\author{
MARKKU NIEMENMAA
}

(Received 12 May 2008)

\begin{abstract}
In this article we show that finite loops with nilpotent inner mapping groups are centrally nilpotent.

2000 Mathematics subject classification: primary 20D10, 20 N05.

Keywords and phrases: centrally nilpotent loop, inner mapping group, connected transversals.
\end{abstract}

\section{Introduction}

Let $Q$ be a groupoid with a neutral element $e$. If each of the two equations $a x=b$ and $y a=b$ has a unique solution for any $a, b \in Q$, then we say that $Q$ is a loop. It should be noted that groups are precisely associative loops. The two mappings $L_{a}(x)=a x$ (left translation) and $R_{a}(x)=x a$ (right translation) are permutations on $Q$ for every $a \in Q$. The permutation group $M(Q)=\left\langle L_{a}, R_{a}: a \in Q\right\rangle$ is called the multiplication group of $Q$. The stabilizer of the neutral element $e$ is denoted by $I(Q)$ and is called the inner mapping group of $Q$. Clearly, $M(Q)$ is transitive on $Q$, and if $Q$ is a group, then $I(Q)=\operatorname{Inn}(Q)$, the group of inner automorphisms of $Q$. Note that $I(Q)=1$ if and only if $Q$ is an abelian group.

A subloop $H$ of $Q$ is normal in $Q$ if $x(y H)=(x y) H,(H x) y=H(x y)$ and $x H=H x$ for any $x, y \in Q$. Normal subloops are naturally kernels of loop homomorphisms. A loop $Q$ is solvable if it has a series $1=Q_{0} \subset \cdots \subset Q_{n}=Q$ where, for each $i, Q_{i-1}$ is a normal subloop of $Q_{i}$ and $Q_{i} / Q_{i-1}$ is an abelian group. If $\alpha \in M(Q)$ and $H \neq Q$ is a nontrivial normal subloop of $Q$, then $\alpha(x H)=\alpha(x) H$ for every $x \in Q$; hence $H$ is a block of $M(Q)$ and $M(Q)$ is imprimitive on $Q$.

In 1996 Vesanen [11] proved the following important result.

THEOREM 1.1. If $Q$ is a finite loop and $M(Q)$ is a solvable group, then $Q$ is a solvable loop.

The centre $Z(Q)$ of a loop $Q$ consists of all elements $a$ which satisfy the conditions $a x=x a,(a x) y=a(x y),(x a) y=x(a y)$ and $(x y) a=x(y a)$ for every $x, y \in Q$. Clearly, $Z(Q)$ is an abelian group, and it is not difficult to show that

(C) 2009 Australian Mathematical Society 0004-9727/2009 \$16.00 
$Z(Q) \cong Z(M(Q))$ (for the details, see [10, Lemma 6.1]). If we write $Z_{0}=1$, $Z_{1}=Z(Q)$ and $Z_{i} / Z_{i-1}=Z\left(Q / Z_{i-1}\right)$, then we obtain a series of normal subloops of $Q$. If $Z_{n-1}$ is a proper subloop of $Q$ and $Z_{n}=Q$, then $Q$ is said to be centrally nilpotent of class $n$. Kepka and Niemenmaa $[6,7,9]$ managed to show that if $Q$ is a finite loop and $I(Q)$ is abelian or a dihedral 2-group, then $Q$ is a centrally nilpotent loop. The purpose of this paper is to show that these results can be generalized in the following way: if $I(Q)$ is a nilpotent group, then $Q$ is a centrally nilpotent loop. An important role in our proof is played by a recent result of Mazur [8] on connected transversals to nilpotent subgroups. We formulate our main result first in terms of group theory by using connected transversals, and then we obtain our loop-theoretical main result as a corollary.

Section 2 contains some basic facts about connected transversals and the proof of our main result. We also present an open problem on the nilpotency class of $Q$ and compare that situation with the one where $I(Q)$ is abelian. In Section 3 we see that certain nilpotent groups are not possible as inner mapping group loops. The proof of the aforementioned result of Mazur depends on the classification of the finite simple groups; in Section 4 we indicate how Mazur's result can be proved without using the classification.

In this paper all groups and loops are finite. For basic facts about loop theory and its connections to group theory, the reader is advised to consult $[2,5,10]$.

\section{Connected transversals and nilpotent inner mapping groups}

Let $G$ be a group and let $H \leq G$. By $H_{G}$ we denote the core of $H$ in $G$ (the largest normal subgroup of $G$ contained in $H$ ). If $A$ and $B$ are two left transversals to $H$ in $G$ and $[A, B] \leq H$, then we say that these two transversals are $H$-connected in $G$. In fact, if $A$ and $B$ are $H$-connected transversals, then $A$ and $B$ are both left and right transversals to $H$ in $G$ [5, Lemma 2.1]. In what follows, we will need the next few results from [5].

Lemma 2.1. Let $C \subseteq A \cup B$ and $K=\langle H, C\rangle$. Then $C \subseteq K_{G}$.

LEMMA 2.2. If $H_{G}=1$, then $N_{G}(H)=H \times Z(G)$.

THEOREM 2.3. If $H$ is cyclic and $G=\langle A, B\rangle$, then $G^{\prime} \leq H$.

For the proofs, see [5, Lemma 2.5, Proposition 2.7 and Theorem 3.5].

Connected transversals appear in loop theory in the following way: if $A=\left\{L_{a} \mid a\right.$ $\in Q\}$ and $B=\left\{R_{a} \mid a \in Q\right\}$, then $A$ and $B$ are $I(Q)$-connected transversals in $M(Q)$. As $M(Q)$ is transitive on $Q$, it follows that the core of $I(Q)$ in $M(Q)$ is trivial. The following characterization theorem was proved by Kepka and Niemenmaa [5, Theorem 4.1] in 1990.

THEOREM 2.4. A group $G$ is isomorphic to the multiplication group of a loop if and only if there exist a subgroup $H$ of $G$ satisfying $H_{G}=1$ and $H$-connected transversals $A$ and $B$ such that $G=\langle A, B\rangle$. 
It should be noted that in the role of the loop elements we have the left cosets of $H$, and the subgroup $H$ is isomorphic to the inner mapping group of the loop. All of the results mentioned in the introduction (on the relation between the structures of $M(Q), I(Q)$ and $Q$ ) were proved by means of group-theoretical arguments and connected transversals. A recent result which is crucial to our investigation is one due to Mazur [8].

THEOREM 2.5. Let $H$ be a nilpotent subgroup of a finite group $G$ which has $H$ connected transversals. Then $G$ is solvable.

The following lemma is a generalized version of the Frattini argument.

LEMMA 2.6. If $K$ is a finite normal solvable subgroup of a group $G$ and $R$ is a Hall subgroup of $K$, then $G=K N_{G}(R)$.

We also need the following lemma in the proof of our main theorem.

LEMMA 2.7. If $Q$ is a finite loop, $I(Q)$ is nilpotent and $I(Q)$ is a maximal subgroup of $M(Q)$, then $Q$ is a cyclic group of prime order and $I(Q)=1$.

Proof. By Theorem 2.5, $M(Q)$ is solvable. From Theorem 1.1 it follows that $Q$ is a solvable loop. If $N \neq Q$ is a nontrivial normal subloop of $Q$, then $M(Q)$ is imprimitive on $Q$ and $I(Q)$ as a one-point stabilizer is not a maximal subgroup of $M(Q)$. Thus we may conclude that $Q$ is both simple and solvable, and hence is a cyclic group of prime order. It is then also clear that $I(Q)=1$.

THEOREM 2.8. Let $H$ be a nilpotent subgroup of a finite group $G$ and assume that there exist $H$-connected transversals $A$ and $B$ in $G$. If $G=\langle A, B\rangle$, then $H$ is subnormal in $G$.

ProOf. Let $G$ be a minimal counterexample. If $H_{G}>1$, then $H / H_{G}$ is subnormal in $G / H_{G}$ and our claim is true. Thus we may assume that $H_{G}=1$. By Theorem 2.4, $G \cong M(Q)$ and $H \cong I(Q)$ for some loop $Q$.

If $H$ is a maximal subgroup of $G$, then by Lemma 2.7, $H \cong I(Q)=1$ and $H$ is subnormal in $G$. We may thus assume that $H$ is not maximal in $G$. By Theorem 2.5, $G$ is a solvable group. If $T<G$ and $H$ is a maximal subgroup of $T$, then $T_{G}>1$ by Lemma 2.1. Now $H T_{G} / T_{G}=T / T_{G}$ is nilpotent, and by induction $T / T_{G}$ is subnormal in $G / T_{G}$; hence $T$ is subnormal in $G$. This naturally means that $N_{G}(H)=H$. Let $N \leq T_{G}$ be a minimal normal subgroup of $G$. As $G$ is solvable, $N$ is an elementary abelian $p$-group. Clearly, $T=N H$. We write $H=P R$, where $P$ is a Sylow $p$ subgroup of $H, R$ is a Hall subgroup of $H$ and $P \cap R=1$. As $N_{G}(H)=H$, it follows that $N_{T}(R)=H$. Since $T$ is subnormal in $G$, we have a subgroup $F>T$ such that $T$ is normal in $F$. If $N_{F}(R)>H$, then we have a subgroup $L \leq N_{F}(R)$ such that $H$ is maximal in $L$ and $L \neq T$. Clearly, $L$ is subnormal in $G$ and then $H=L \cap T$ is subnormal in $G$. Thus we may assume that $N_{F}(R)=H$. Now $R$ is a 
Hall subgroup of $T$, so we can apply Lemma 2.6 and get $F=T N_{F}(R)=T H=T$, which is a contradiction. We therefore conclude that $H$ is subnormal in $G$.

REMARK. The mapping $f: I(Q) \rightarrow I(Q / Z(Q))$ defined by

$$
f(\alpha)(x Z(Q))=\alpha(x) Z(Q)
$$

is a surjective homomorphism, and

$$
K=\operatorname{Ker}(f)=\{\alpha \in I(Q) \mid \alpha(x) Z(Q)=x Z(Q) \forall x \in Q\}
$$

(For the details, see [2, pp. 279-280].)

COROLlary 2.9. If $Q$ is a finite loop and $I(Q)$ is nilpotent, then $Q$ is a centrally nilpotent loop.

Proof. By Theorem 2.8 and Lemma 2.2, $Z(M(Q))>1$ and thus $Z(Q)>1$. If $K$ is as in the preceding remark, then we have $I(Q / Z(Q)) \cong I(Q) / K$. We conclude that $Z(Q / Z(Q))>1$. We may continue in this manner, and it follows that $Q$ is centrally nilpotent.

Now it is quite natural to pose the following question.

PROBLEM. Let $I(Q)$ be a nilpotent group of class $n$. What can we say about the nilpotency class of the loop $Q$ ?

We wish to point out that, even in the case where $I(Q)$ is a finite abelian group, it is not clear how the nilpotency class of $Q$ is determined. If $p$ is a prime number and $I(Q) \cong C_{p} \times C_{p}$ or $I(Q) \cong C_{p} \times C_{p} \times C_{p}$, then $Q$ is centrally nilpotent of class two (for the details, see [3, 10]). In 2007, Csörgö [4] constructed an example of a loop $Q$ of order $2^{7}$ with an elementary abelian $I(Q)$ of order $2^{6}$ and with nilpotency class three. It is not known if there exist loops with abelian inner mapping groups and with nilpotency class four.

\section{Loop capable nilpotent groups}

Groups that are isomorphic to inner automorphism groups of groups are called capable groups. We shall call groups which are isomorphic to inner mapping groups of loops loop capable groups, and we will investigate the following problem: which nilpotent groups are (or are not) loop capable groups? We shall next show that if at least one of the primary components of a finite nilpotent group $H$ is cyclic, then $H$ is not a loop capable group. We assume that $A$ and $B$ are $H$-connected transversals.

THEOREM 3.1. Let $H \cong C \times T$, where $C>1$ is cyclic, $T$ is nilpotent and $\operatorname{gcd}(|C|,|T|)=1$. If $G=\langle A, B\rangle$, then $H_{G}>1$. 
Proof. Let $G$ be a minimal counterexample and hence assume that $H_{G}=1$. By Theorem 2.3, we may assume that $T>1$. From Lemma 2.2 and Theorem 2.8 it follows that $Z(G)>1$. Take $z \in Z(G)$ such that $|z|=p$, where $p$ is a prime number; then consider the factor groups $G /\langle z\rangle$ and $H\langle z\rangle /\langle z\rangle$. Since $G$ is a minimal counterexample, there is a normal subgroup $K$ of $G$ such that $\langle z\rangle<K \leq H\langle z\rangle$ and $C \leq K$. Thus $|K|=|C| t p$, where $t$ divides $|T|$. As Sylow subgroups of $K$ are normal in $G$ and $H_{G}=1$, we may conclude that $t=1, K=C \times\langle z\rangle$ and $C$ is a $p$-group.

Since $H$ is nilpotent, it follows from Theorem 2.5 that $G$ is solvable. This guarantees the existence of Hall subgroups. Let $\Pi$ be a set which contains exactly those prime numbers that divide $|T|$, and let $S \geq T$ be a Hall $\Pi$-subgroup of $G$; then write $F=K N_{G}(S)$. If $E \geq F$ is a maximal subgroup of $G$, then $E>H$ and, by Lemma 2.1, $E_{G}>1$. By Theorem 2.8, $H E_{G} / E_{G}=E / E_{G}$ is subnormal in $G / E_{G}$; hence $E$ is subnormal and, in fact, normal in $G$. By using the generalized Frattini argument, we get $G=E N_{G}(S)=E$, which is a contradiction. Thus $F=G$.

If $g \in G$, then $g=k n$, where $k \in K$ and $n \in N_{G}(S)$. Now $K \leq C_{G}(T)$ and thus $T^{g}=T^{k n}=T^{n} \leq S^{n}=S$. Since $M=\left\langle T^{g}: g \in G\right\rangle$ is normal in $G$ and $T \leq M \leq S$, it follows that $H M / M$ is cyclic. By Theorem 2.3, $(G / M)^{\prime} \leq H M / M$ and hence $G^{\prime} \leq H M$. Therefore $H M$ is normal in $G$ and $H M \cap K=C$ is normal in $G$. But this clearly contradicts the assumption that $H_{G}=1$, so the proof is complete.

Upon combining Theorem 3.1 with Theorem 2.4, we get the following result.

Corollary 3.2. Let $H$ be as in Theorem 3.1. Then $H$ is not a loop capable group.

In the case of finite nonnilpotent groups, we formulate the following conjecture.

CONJeCture. Assume that $H$ is a finite nonnilpotent group which is loop capable. Then $H \times C$, where $C$ is a nontrivial finite cyclic group such that $\operatorname{gcd}(|H|,|C|)=1$, is not loop capable.

\section{Some remarks}

In the proof of Theorem 2.5 (that is, [8, Theorem 1]), Mazur starts with the assumption that $G$ is a minimal counterexample. Thus $G$ is a nonsolvable finite group with a nilpotent subgroup $H$ and $H$-connected transversals $A$ and $B$. As in [8, Section 2], it then follows that $H$ is a maximal subgroup of $G$ and $G=D H$, where $D$ is a minimal normal subgroup of $G$. Furthermore, $H$ is a Sylow 2-subgroup of $G$ and $D$ is a nonabelian simple group. After this, in [8, Section 3], the classification of finite simple groups is used to conclude that $D \cong P S L(2, q)$, where $q=2^{n} \pm 1 \geq 7$ is a prime number.

We can, however, avoid the use of the classification by applying the following result of Baumann [1] from 1976 (we follow the original article written in German).

THEOREM 4.1. Let $G$ be a nonsolvable finite group with a nilpotent maximal subgroup. Then $O^{2}(G / F(G))$ is a direct product of simple groups whose Sylow 2-subgroups are dihedral. 
Here $O^{2}(X)$ denotes the smallest normal subgroup with a factor group which is a 2-group, and $F(X)$ is the largest nilpotent normal subgroup of $X$. The simple groups which are the components of the direct product are isomorphic to $\operatorname{PSL}(2, q)$, where $q=2^{n} \pm 1 \geq 7$ is a prime number or $q=9$. The application of Baumann's theorem therefore gives us directly the structure of $D$ (the $q=9$ case does not cause any problems). We may then proceed as in [8, Section 4], and the solvability of $G$ follows.

\section{References}

[1] B. Baumann, 'Endliche nichtauflösbare Gruppen mit einer nilpotenten maximalen Untergruppe', J. Algebra 38 (1976), 119-135.

[2] R. H. Bruck, 'Contributions to the theory of loops', Trans. Amer. Math. Soc. 60 (1946), 245-354.

[3] P. Csörgö, 'On connected transversals to abelian subgroups and loop theoretical consequences', Arch. Math. (Basel) 86(6) (2006), 499-516.

[4] , 'Abelian inner mappings and nilpotency class greater than two', European J. Combin. 28(3) (2007), 858-867.

[5] T. Kepka and M. Niemenmaa, 'On multiplication groups of loops', J. Algebra 135 (1990), $112-122$.

[6] _ 'On connected transversals to abelian subgroups in finite groups', Bull. London Math. Soc. 24 (1992), 343-346.

[7] _ 'On connected transversals to abelian subgroups', Bull. Aust. Math. Soc. 49 (1994), $121-128$.

[8] M. Mazur, 'Connected transversals to nilpotent groups', J. Group Theory 10 (2007), 195-203.

[9] M. Niemenmaa, 'On loops which have dihedral 2-groups as inner mapping groups', Bull. Aust. Math. Soc. 52 (1995), 153-160.

[10] M. Niemenmaa and M. Rytty, 'Connected transversals and multiplication groups of loops', Quasigroups Related Systems 15 (2007), 95-107.

[11] A. Vesanen, 'On solvable loops and groups', J. Algebra 180 (1996), 862-876.

\section{MARKKU NIEMENMAA, Department of Mathematical Sciences, University of Oulu, PL 3000, 90014 Oulu, Finland \\ e-mail: mniemen@cc.oulu.fi}

